\title{
Long-term Existence of Donor Adipose-derived Stem Cells in the Recipient May Modulate the Vascularized Composite Tissue Allotransplantation Survival in a Rodent Model
}

\author{
Chien-Chang Chen ${ }^{1,2}$, Shigeru Goto ${ }^{3}$, Chia-Chun Tsai ${ }^{1,2}$ and Yur-Ren Kuo ${ }^{1,2 *}$ \\ ${ }^{1}$ Department of Plastic and Reconstructive Surgery, Kaohsiung Chang Gung Memorial Hospital and Chang Gung University College of Medicine, Taiwan \\ ${ }^{2}$ Vascularized Composite Tissue Allotransplantation Center, Kaohsiung Chang Gung Memorial Hospital and Chang Gung University College of Medicine, Taiwan \\ ${ }^{3}$ Liver Transplantation Program and Department of Surgery, Kaohsiung Chang Gung Memorial Hospital and Chang Gung University College of Medicine, Taiwan
}

\begin{abstract}
Our previous studies have demonstrated donor adipose-derived stem cells (ASCs) combined with short-term immunosuppressant therapy could prolong vascularized composite tissue allotransplantation (VCA) survival in a rodent hind-limb model. In this study, we investigated whether the homing and migration of donor ASCs may modulate VCA survival. Orthotopic hind-limb transplants from male Wistar to Lewis rats were performed (day 0). Donor ASCs were propagated from the adipose-tissue and subculture originated from green fluorescent protein (GFP) transgenic Wistar rats. The transplanted rat (GFP-negative Lewis) received the immunosuppressive protocol similar to our previous design including short-term cyclosporine-A (CsA, days $0-+20)$, anti-lymphocyte serum (ALS; $0.5 \mathrm{ml} \mathrm{i.p.;} \mathrm{-4,}$ $+1)$, and three rounds of GFP+-ASCs $(2 \times 106$ cells/time, i.v. on days $+1,+7$, and +21$)$. The engraftment assessment of various donor and recipient tissues were performed by using immuno-fluorescent staining. Flow cytometry was performed to quantify the GFP+-ASCs. The results revealed allotransplant survival was significantly prolonged (>100 days) exclusively in ASC-ALS-CsA group which was well correlated with long-term existence of donor GFP-positive ASCs in the recipient. Flow cytometric analysis also revealed GFP+-ASCs apparent expression in the recipient peripheral blood in 2 weeks post-ASC infusion but skewing decrease post-transplantation. The immunofluorscent staining performed on tissue biopsy samples revealed the GFP positive cells existed in the alloskin and recipient skin and liver and spleen parenchymal tissues 2 weeks post-ASC injection. However, no apparent expressions of GFP+-ASCs were found in recipient tissues at 16 weeks post-transplantation except in recipient spleen parenchyma. These indicated donor ASCs long-term existed in the recipient spleen tissue and circulating blood may lead to prolong allograft survival.
\end{abstract}

Keywords: Vascularized composite tissue allotransplantation; Adipose-derived stem cells; Engraftment

\section{Introduction}

Recent advances in vascularized composite tissue allotransplantation (VCA) represent the essence of reconstructive surgery [1]. Indeed, a successful human hand and partial face allotransplantation have been performed [2,3]. However, VCA transplants are not routinely performed for tissue reconstruction because lifelong administrations of immunosuppressive agents have potentially harmful side effects $[4,5]$. Furthermore, even if a patient has excellent compliance, conventional immunosuppressive protocols may not be sufficient to prevent chronic rejection. Consequently, researchers have been eagerly seeking alternative methods for establishing lifelong tolerance while minimizing toxicity $[6,7]$.

Mesenchymal stem cells (MSCs) are multipotent and nonhematopoietic progenitor cells in the adult bone marrow and are capable of differentiating into various mesenchymal cell types [8]. Previous studies have demonstrated that MSCs do not express major histocompatibility complex (MHC) class II or co-stimulatory molecules, such as B7-1, B7-2, or CD40. Therefore, they are most likely unable to stimulate allo-specific $\mathrm{T}$ cells proliferation $[9,10]$. Studies revealed that multiple rounds of donor bone marrow-derived MSCs infusion therapies combined with bone marrow transplantation and short-term immunosuppressant could modulate graft-versushost disease and prevent graft rejection in a swine hind-limb VCA model [11]. We demonstrated that administration of donor MSCs without BMT increased allotransplant survival, which may correlate with an increase in regulatory $\mathrm{T}$ cell populations [12]. However, the preparation of bone marrow-derived MSCs was time consuming. Adipose-derived mesenchymal stem cells (ASCs) have been found to share characteristics, including multipotency with bone marrowderived MSCs $[13,14]$. Additionally, harvesting adipose tissue to obtain ASCs is easier than preparing MSCs from bone marrow and has greater potential for clinical applications [15]. Our previous study showed multiple rounds of donor ASCs infusion therapies combined with short-term anti-lymphocyte serum (ALS, administered 4 days before and 1 day after transplantation) and cyclosporine-A (CsA, day 0-20 post-transplantation) prolonged VCA survival in a rodent (BrownNorway to Lewis rat) hind-limb VCA model. ASCs alone or shortterm of ALS/CsA is also not enough to prevent VCA rejection [16]. Furthermore, the results demonstrated ASCs have immunomodulatory abilities to regulate $\mathrm{T}$ cell function, modulate soluble cytokine expression, and prolong allotransplant survival $[7,16]$. These results

${ }^{*}$ Corresponding author: Yur-Ren Kuo, MD, PhD, Department of Plastic and Reconstructive Surgery, Kaohsiung Chang Gung Memorial Hospital, 123 Ta-Pei Road, Niao-Sung, Kaohsiung 83305, Taiwan, Tel: 886-7-7317123, ext. 8002; Fax: 886-7-7311696; E-mail: t1207816@ms22.hinet.net; kuoyr@adm.cgmh.org.tw

Received December 01, 2013; Accepted December 18, 2013; Published December 20, 2013

Citation: Chen CC, Goto S, Tsai CC, Kuo YR (2013) Long-term Existence of Donor Adipose-derived Stem Cells in the Recipient May Modulate the Vascularized Composite Tissue Allotransplantation Survival in a Rodent Model. J Stem Cell Res Ther S6: 005. doi:10.4172/2157-7633.S6-005

Copyright: ( 2013 Chen CC, et al. This is an open-access article distributed under the terms of the Creative Commons Attribution License, which permits unrestricted use, distribution, and reproduction in any medium, provided the original author and source are credited. 
Citation: Chen CC, Goto S, Tsai CC, Kuo YR (2013) Long-term Existence of Donor Adipose-derived Stem Cells in the Recipient May Modulate the Vascularized Composite Tissue Allotransplantation Survival in a Rodent Model. J Stem Cell Res Ther S6: 005. doi:10.4172/2157-7633. S6-005

Page 2 of 5

indicated the protective and promotional effects in combination of immunosuppressant needs for ASCs to migrate and homing into the graft, which may eventually lead to prolong the allograft. Nevertheless, the fate of donor ASCs after composite allografting that survives for long periods is still unclear. Therefore, in this study, we explore the distribution and role of donor ASCs after VCA by using the green fluorescent protein (GFP) transgenic rats.

\section{Materials and Methods}

\section{Animals}

Twelve male Lewis (LEW, RT1 ${ }^{1}$ ) rats aged 8-12 weeks and weighing 240-350 g were used as recipients. Orthotopic hind-limb transplants from male Wistar without green fluorescent protein (GFP) expression to Lewis rats were performed (day 0). The Wistar and Lewis rats were purchased from the National Laboratory Animal Breeding and Research Center, Taiwan. ASCs were propagated from the donor adipose-tissue and subculture originated from green fluorescent protein (GFP) transgenic Wistar rats. GFP-transgenic Wistar rats [W-Tg (Per1luc)10a], which sponsored from Osaka university, Japan (Prof. Tsutomu Kurosawa), and are band bred in the Experimental Animal Center of Kaohsiung Chang Gung Memorial Hospital. The animals are maintained under standard conditions with access to rodent chow and water. They were all treated humanely, according to the guidelines provided in the Guide for the Care and Use of Laboratory Animals, published by the National Institutes of Health. The Division of Laboratory Animal Resources at Kaohsiung Chang Gung Memorial Hospital (KCGMH) provided veterinary care to the rodents. The experiments conducted were approved by the Institutional Animal Care and Use Committee (IACUC) at KCGMH.

\section{Culture of $\mathrm{GFP}^{+}$-ASCs}

$\mathrm{GFP}^{+}$-ASCs were propagated from the adipose-tissue of GFPtransgenic Wistar and subculture. The isolation of $\mathrm{GFP}^{+}$-ASCs was performed according to published methodologies [16]. Groin adipose tissue was minced, washed, and digested with $0.2 \%$ collagenase type I for $30 \mathrm{~min}$ at $37^{\circ} \mathrm{C}$. The suspension was centrifuged at $500 \times \mathrm{g}$ for $5 \mathrm{~min}$ at room temperature to pellet cells from the stromal vascular fraction. Following RBC lysis with $0.83 \% \mathrm{NH}_{4} \mathrm{Cl}$, the cells were washed with $\mathrm{PBS}$, resuspended in maintenance medium, and plated in culture dishes for $4 \mathrm{~h}$. The non-adherent cells were removed, and the attached cells were considered to be P0 ASCs. ASCs were cultured in maintenance medium consisting of DMEM supplemented with $10 \%$ fetal bovine serum and $1 \%$ penicillin-streptomycin at $37^{\circ} \mathrm{C}$ in a humidified with $5 \% \mathrm{CO}_{2}$, and subcultured prior to confluency. The ASCs were characterized by flow cytometry following positive surface staining for CD44, CD90, MHC-I, and CD106; but not for CD45, and MHC-II. ASCs were tested as our previously described procedures for their ability to differentiate into various mesenchymal lineages, including adipocytes, osteoblasts, and chondrocytes [16].

\section{Orthotopic hind-limb vascularized composite tissue allotransplantation model}

Hind-limb transplantations were performed on the rats according to our previously described procedures [17]. The hind limbs of the donor Wistar rats without GFP expression were amputated at the mid-thigh level and placed in gauze soaked with cold normal saline. The hind limbs of the recipient LEW rats were amputated in a similar manner at the mid-thigh level, following preparation of the recipient vessels. The donor and recipient femurs were then joined by performing osteosynthesis, using an 18-gauge needle as the intramedullary rod.
This method was followed by a repair of the ventral and dorsal muscle groups with 4-0 interrupted silk sutures. The sciatic nerve was repaired with 9-0 nylon sutures. The femoral vein and artery were anastomosed with 10-0 nylon sutures under a stereomicroscope (Zeiss ${ }^{\circledR}$, Germany). The skin was stitched together with 4-0 silk sutures.

\section{Animal experimental design}

Twelve orthotopic hind-limb VCA from Wistar to Lewis rats were performed on day 0. Each subgroup included 6 rats. Group 1 (control group) did not receive any treatment. In Group II, transplanted rat was treated with a combination of CsA (days 0-20), ALS (administered 4 days before and 1 day after CTA), and donor ASCs $\left(2 \times 10^{6}\right.$ cells/ time on days 1,7 , and 21 after CTA) in combination. The dosage and protocol of ASCs was mimicked from our previous study [16]. Graft rejection was assessed daily.

\section{Flow cytometry assessment}

To determine whether donor $\mathrm{GFP}^{+}$-ASC cells migrated into recipient peripheral blood, flow cytometric analysis was performed one week, two weeks, and 16 weeks after ASCs infusion. The cells were incubated with $5 \mu \mathrm{L}$ of polyclonal antibody against GFP (Abcam, Cambridge, UK) to identify infusioned donor ASCs. After incubation for $30 \mathrm{~min}$ at room temperature, the cells were centrifuged, washed, and analyzed on a flow cytometer (Becton Dickinson, CA). Data were subsequently analyzed using the BD FACScan TM program.

\section{Immunohistochemical (IHC) staining}

Frozen sections were obtained from the allo-skin of the grafted limbs, recipient skin, spleen parenchyme, and liver parenchymal tissue on 2 weeks and 16 weeks after the GFP+-ASC infusion. Samples were embedded in Tissue-Tek ${ }^{\circledR}$ OTC (Sakura Fine Technical Co., Ltd.) and stored at $-20^{\circ}$ until use. Cryo-sections at $10 \mu \mathrm{m}$ of thickness were adhered to poly-L-lysine-coated glass cover-slips and fixed with acetone for $10 \mathrm{~min}$. After polyclonal GFP-antibody (Abcam, Cambridge, UK) labeling, these sections were immediately observed for GFP expression by fluorescent microscopy. The sections were also stained with Hematoxylin-Eosin (H\&E) according to standard procedures. Photographs of GFP-expressing sections and H\&E sections were composed using Photoshop software (Adobe Systems, Japan). The morphologic changes in the GFP-positive tissue component were examined. For semi-quantification of immunofluorscent immunostaining, the tissue sections were analyzed. Four randomly selected areas were then photographed at $400 \times$ magnification. All images were captured using a Cool CCD camera. The images were analyzed using the Image-Pro Plus image analysis software (Media Cybernetics, Silverbernetics Spring, MD).

\section{Results}

\section{Donor ASCs combined with short-term ALS-CsA prolonged VCA survival}

The allotransplanted limbs were rejected between days 8 and 14 post-transplantation in the controls. Recipients treated with multiple dosages of donor ASCs in addition to short-term ALS/CsA treatment resulted in significant increases in allograft survival ( $>100$ days) of recipients compared to control group (Table 1).

\section{$\mathrm{GFP}^{+}$-ASC existed in recipient circulating blood but skewed decrease}

Flow cytometric analysis of recipient peripheral blood revealed 
Citation: Chen CC, Goto S, Tsai CC, Kuo YR (2013) Long-term Existence of Donor Adipose-derived Stem Cells in the Recipient May Modulate the Vascularized Composite Tissue Allotransplantation Survival in a Rodent Model. J Stem Cell Res Ther S6: 005. doi:10.4172/2157-7633. S6-005

Page 3 of 5

that $\mathrm{GFP}^{+}$-ASC cell populations were significantly increased at one week and two weeks post-transplant in animals treated with $\mathrm{GFP}^{+} \mathrm{ASCs}$ and short-term CsA and ALS, compared to the controls. However, the percentage of $\mathrm{ASC}_{-} \mathrm{GFP}^{+}$cell expressions in the recipient peripheral blood skewed decrease post-transplantation. The $\mathrm{GFP}^{+}$-ASC cell expressions in recipient circulating blood still existed at 16 weeks posttransplant in rats treated with $\mathrm{GFP}^{+}$-ASCs, CsA and ALS (Figure 1).

\section{$\mathrm{GFP}^{+}$-ASC expressed in recipient and donor tissues post-VCA}

The immunofluorscent staining performed on tissue biopsy samples revealed the GFP positive cells existed in the alloskin and recipient skin and liver and spleen parenchymal tissues 2 weeks post-ASC injection (Figure 2). No apparent expressions of ASC-GFP positive cells were found in recipient tissues at 16 weeks post-transplantation except spleen parenchymal tissue still retain GFP positive cells (Figure 3). These results indicated donor ASCs could be long-term existence in the recipient spleen and might play an immunomodulator to prolong allograft survival.

\begin{tabular}{|l|c|c|c|c|}
\hline Group & $\begin{array}{c}\text { ALS } \\
\mathbf{( - 4 , + 1 d )}\end{array}$ & $\begin{array}{c}\text { CsA } \\
\mathbf{( 0 - 2 0 d})\end{array}$ & $\begin{array}{c}\text { GFP }^{+} \text {-ASC } \\
\mathbf{( + 1 , + 7 , + 2 1 d})\end{array}$ & Allograft survival day \\
\hline 1 & - & - & - & $9,9,11,8,10,14$ \\
\hline 2 & - & - & - & $105^{\star},>150,>150,>150,>150,>150$ \\
\hline
\end{tabular}

*Expired during sampling, but no rejection of allograft

Table 1: Analysis of allo-transplant survival days in a rodent hind-limb VCA model.

\section{Discussion}

Our previous study indicated multiple round of donor adipose-derived stem cells (ASC) in combination with transient immunosuppressant (ALS/CsA) significantly prolonged VCA survival and facilitate long-term graft acceptance in a rodent hind-limb VCA model (from Brown-Norway to Lewis inbred strain) [16]. In this study, we mimicked our previous protocol to trace the donor ASCs engraftment. However, no green fluorescent protein (GFP) transgenic $\mathrm{BN}$ rats were available. Therefore, in this study, ASCs combined with short term immunosuppressant were applied in different rodent model (Wistar as a donor to Lewis strain). The $\mathrm{GFP}^{+}$-ASCs were propagated from the adipose-tissue of GFP-transgenic Wistar and subculture. The results revealed the multiple rounds of ASCs (days 6, 8, 10 posttransplantation) in addition to short-term ALS/CsA treatment indeed resulted in significant increases in allograft survival as compared to that in controls.

Although the bio-mechanisms as to how ASCs involved modulation of anti-inflammatory cytokine expression and $\mathrm{T}$ cell subsets have been proposed $[16,18]$ the fate of donor ASCs after composite allografting that survives for long periods is still unclear. Therefore, the distribution of donor ASCs engraftment after VCA by using the GFP transgenic rats has been investigated.

Studies demonstrated ASC could modulate regulatory T-cells in vitro and in vivo [19]. Our study indicated CD4+/CD25+/Foxp3+
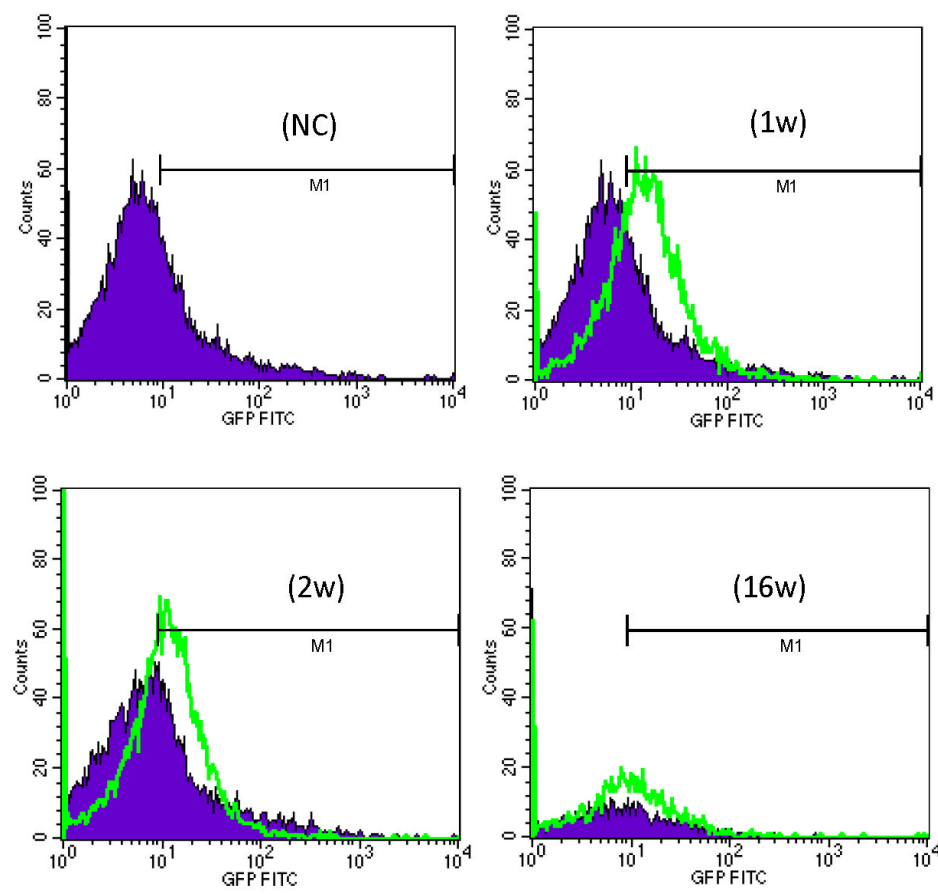

\section{Wistar-GFP ${ }^{+}$ASCs}
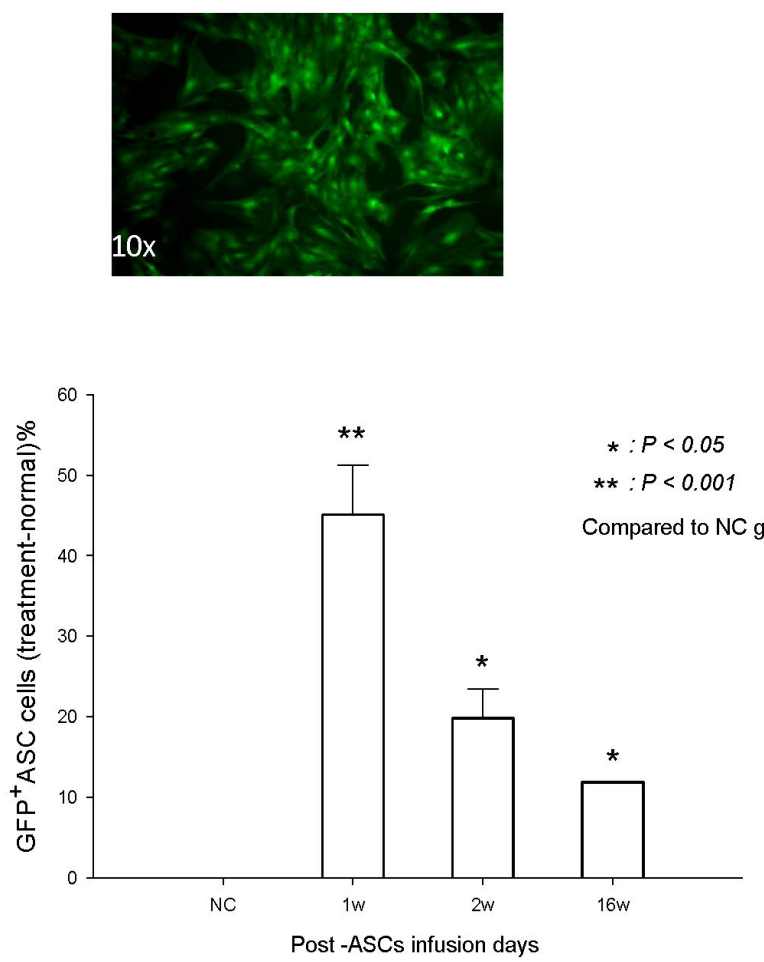

Figure 1: Flow cytometric analysis of recipient peripheral blood revealed that GFP+-ASC cell populations were significantly increased at one week and two weeks post-transplant in animals treated with GFP ${ }^{+}$ASCs and short-term CsA-ALS, compared to the controls. The GFP+-ASC cell expressions in recipient circulating blood still existed at 16 weeks post-transplant in rats treated with GFP+-ASCs, CsA and ALS, although skewing decrease post-transplantation. The indicated signals were statistically significant $(P<0.05)$. 
Citation: Chen CC, Goto S, Tsai CC, Kuo YR (2013) Long-term Existence of Donor Adipose-derived Stem Cells in the Recipient May Modulate the Vascularized Composite Tissue Allotransplantation Survival in a Rodent Model. J Stem Cell Res Ther S6: 005. doi:10.4172/2157-7633. S6-005

Page 4 of 5

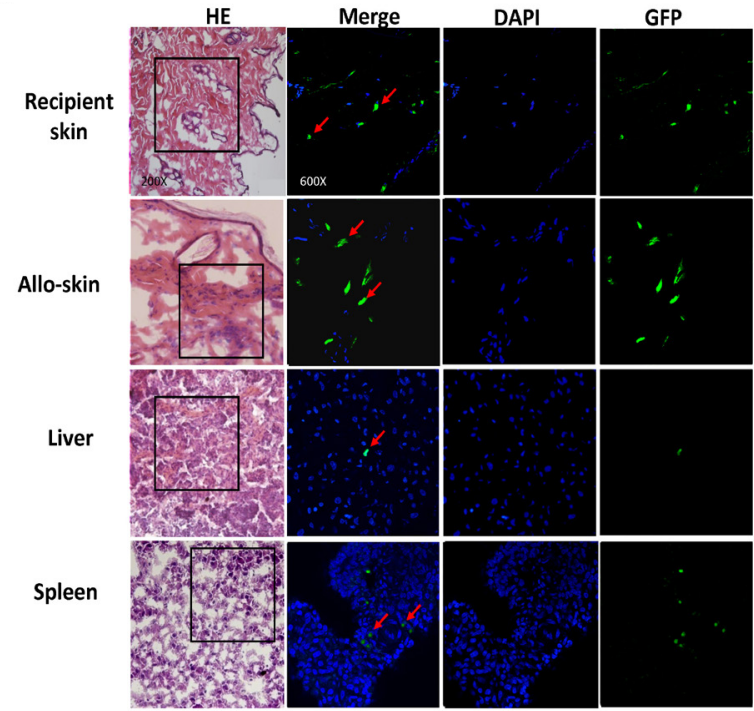

B

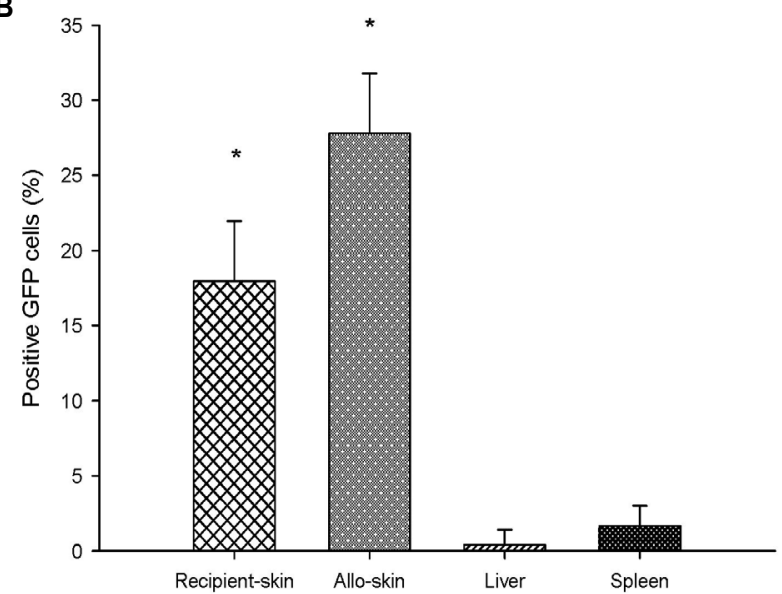

Figure 2: ((A): The immunofluorscent staining performed on tissue biopsy samples revealed the GFP positive cells existed in the alloskin and recipient skin and recipient liver and spleen parenchymal tissues 2 weeks post-ASC injection. (B): GFP positive cells in different immunofluorscent staining tissues were counted by using semi-quantitative analysis. Abbreviations: GFP: Green Fluorescent Protein; HE: Hematoxylin-Eosin; and DAPI: 4',6-diamidino-2-phenylindole.

$\mathrm{T}$ cells increased significantly at early time points post-transplant in animals treated with donor ASCs, short term CsA and ALS compared to untreated controls in a rodent hind-limb VCA model [16]. IHC staining revealed significant numbers of Foxp3+ T cells in allo-skin biopsies from animals treated with donor ASC-CsA-ALS compared to the other groups [16]. These results demonstrate that donor ASCs are a promising therapy that could help to lessen rejection of allotransplantation, which is correlated with regulation of $\mathrm{T}$ cells.

To investigate the fate of ASC after infusion to recipient, flow cytometry were analyzed the expressions of GFP+-ASCs in the circulating blood. The results revealed $\mathrm{GFP}^{+}$-ASCs apparently transient expression in the recipient peripheral blood in 2 weeks postASC infusion but skewing decrease. However, in blood even after 16 weeks post-transplantation, GFP positive graft ASC cells can still be detected. This evidence indicated the donor $\mathrm{GFP}^{+}$-ASCs cell could
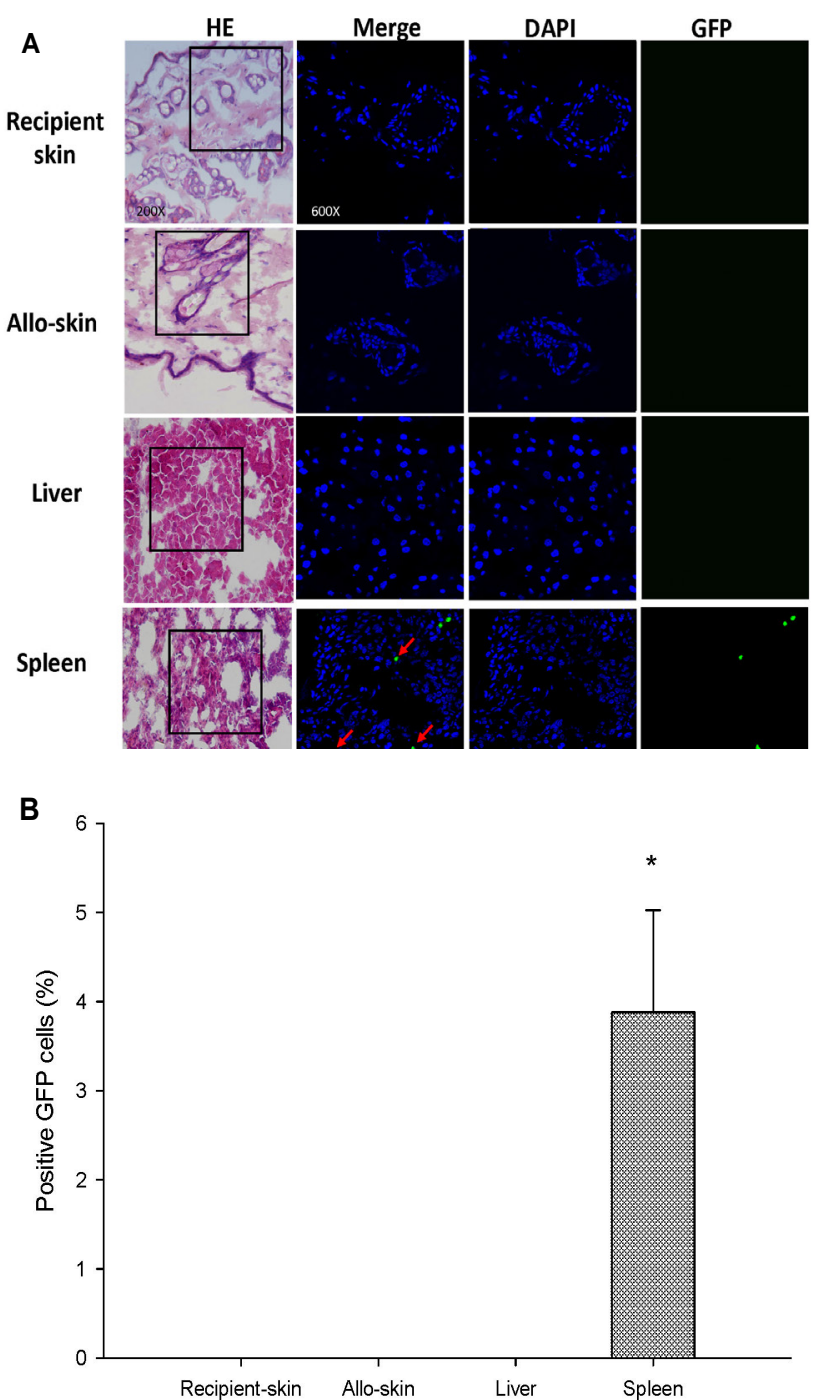

Figure 3: (A) The immunofluorscent staining performed on tissue biopsy samples revealed no apparent expressions of ASC-GFP+ cells were found in recipient tissues at 16 weeks post-transplantation except spleen parenchymal tissue. These indicated donor ASCs could be long-term existence in the recipient spleen. (B) Semi-quantitative analyses of GFP positive cells in different immunofluorscent staining tissues were counted. The indicated signals were statistically significant $(P<0.05)$. Abbreviations: GFP: Green Fluorescent Protein; HE: Hematoxylin-Eosin; and DAPI: 4',6-Diamidino-2-Phenylindole.

exist in recipient circulating blood. In contrast, the immunofluorscent staining performed on tissue biopsy samples revealed the GFP positive cells existed in the alloskin and recipient skin, and recipient liver and spleen parenchymal tissues 2 weeks post-ASC injection. No apparent expressions of $\mathrm{GFP}^{+}$-ASCs were found in recipient skin and liver tissues at 16 weeks post-transplantation. However, in solid tissues, only recipient spleen parenchyma were existed the GFP positive cells at 16 weeks post-transplantation. This indicated recipient spleen parenchyma retained donor GFP positive cells and limited long-term survival of the graft cells.

Spleen is the most important lymphoid organ to modulate immune cells and function. Our results revealed the donor stem cells could 
Citation: Chen CC, Goto S, Tsai CC, Kuo YR (2013) Long-term Existence of Donor Adipose-derived Stem Cells in the Recipient May Modulate the Vascularized Composite Tissue Allotransplantation Survival in a Rodent Model. J Stem Cell Res Ther S6: 005. doi:10.4172/2157-7633. S6-005

Page 5 of 5

engraft in recipient spleen parenchyma. This evidence indicated at least in part donor ASCs exist in the recipient tissue and some might transform to mesenchyme tissue in the recipient spleen as an immunomulator to modulate immune cells regulation after VCA. Therefore, we propose the reason of donor ASCs prolongation allograft survival might through educate the naïve immune cells and regulate their function to decrease the allograft rejection. However, in this study, the long-term results between donor cells and recipient cells redistribution could not be assessed. Although we did not analyze these cells in this study, future studies are needed to address the presence of T cells, B cells and macrophages in the vicinity of the grafted GFP positive cells. Further studies are needed to elucidate the mechanism of mesenchymal stem cells renewal and induction immune modulation.

\section{Acknowledgements}

We would like to thanks Prof. Tsutomu Kurosawa provided GFP-transgenic Wistar rats [W-Tg (Per1-luc)10a], which sponsored from Osaka university, Japan.

\section{Financial Support}

This work was supported in part by Contract No. grant NMRPG886243 form the National Science Council, Taiwan; and No. grant CMRPG8A0051 and CMRPG880543 from the Chang Gung Memorial Hospital Research Project Taiwan.

\section{References}

1. Lee WP (2001) Composite tissue transplantation: more science and patience needed. Plast Reconstr Surg 107: 1066-1070.

2. Jones JW, Gruber SA, Barker JH, Breidenbach WC (2000) Successful hand transplantation. One-year follow-up. Louisville Hand Transplant Team. N Engl J Med 343: 468-473.

3. Dubernard JM, Devauchelle B (2008) Face transplantation. Lancet 372: 603-604.

4. Kaufman CL, Breidenbach W (2011) World experience after more than a decade of clinical hand transplantation: update from the Louisville hand transplant program. Hand Clin 27: 417-421.

5. Madani H, Hettiaratchy S, Clarke A, Butler PE (2008) Immunosuppression in an emerging field of plastic reconstructive surgery: composite tissue allotransplantation. J Plast Reconstr Aesthet Surg 61: 245-249.

6. Ravindra KV, Xu H, Bozulic LD, Song DD, Ildstad ST (2012) The need for inducing tolerance in vascularized composite allotransplantation. Clin Dev Immunol 2012: 438078.
7. Kuo YR, Chen CC, Goto S, Lin PY, Wei FC, et al. (2012) Mesenchymal stem cells as immunomodulators in a vascularized composite allotransplantation. Clin Dev Immunol 2012: 854846.

8. Pittenger MF, Mackay AM, Beck SC, Jaiswal RK, Douglas R, et al. (1999) Multilineage potential of adult human mesenchymal stem cells. Science 284 143-147.

9. Krampera M, Glennie S, Dyson J, Scott D, Laylor R, et al. (2003) Bone marrow mesenchymal stem cells inhibit the response of naive and memory antigenspecific T cells to their cognate peptide. Blood 101: 3722-3729.

10. Glennie S, Soeiro I, Dyson PJ, Lam EW, Dazzi F (2005) Bone marrow mesenchymal stem cells induce division arrest anergy of activated $T$ cells. Blood 105: 2821-2827.

11. Kuo YR, Goto S, Shih HS, Wang FS, Lin CC, et al. (2009) Mesenchyma stem cells prolong composite tissue allotransplant survival in a swine model. Transplantation 87: 1769-1777.

12. Kuo YR, Chen CC, Shih HS, Goto S, Huang CW, et al. (2011) Prolongation of composite tissue allotransplant survival by treatment with bone marrow mesenchymal stem cells is correlated with T-cell regulation in a swine hind-limb model. Plast Reconstr Surg 127: 569-579.

13. Izadpanah R, Trygg C, Patel B, Kriedt C, Dufour J, et al. (2006) Biologic properties of mesenchymal stem cells derived from bone marrow and adipose tissue. J Cell Biochem 99: 1285-1297.

14. Zhu X, Du J, Liu G (2012) The comparison of multilineage differentiation of bone marrow and adipose-derived mesenchymal stem cells. Clin Lab 58: 897-903.

15. Pachon-Pena G, Yu G, Tucker A, Wu X, Vendrell J, et al. (2011) Stromal stem cells from adipose tissue and bone marrow of age-matched female donors display distinct immunophenotypic profiles. J Cell Physiol 226: 843-851.

16. Kuo YR, Chen CC, Goto S, Lee IT, Huang CW, et al. (2011) Modulation of immune response and T-cell regulation by donor adipose-derived stem cells in a rodent hind-limb allotransplant model. Plast Reconstr Surg 128: 661e-672e.

17. Kuo YR, Huang CW, Goto S, Wang CT, Hsu LW, et al. (2009) Alloantigenpulsed host dendritic cells induce T-cell regulation and prolong allograft survival in a rat model of hindlimb allotransplantation. J Surg Res 153: 317-335.

18. Gonzalez-Rey E, Gonzalez MA, Varela N, O'Valle F, Hernandez-Cortes P, et al. (2010) Adipose-derived mesenchymal stem cells reduce inflammatory and T cell responses and induce regulatory $T$ cells in vitro in rheumatoid arthritis. Ann Rheum Dis 69: 241-248.

19. Mizuno H (2010) Adipose-derived stem and stromal cells for cell-based therapy: current status of preclinical studies and clinical trials. Curr Opin Mol Ther 12 442-449.
This article was originally published in a special issue, Transplantation Immunology handled by Editor(s). Qing Ma, University of Texas, USA 